\title{
Minimum inhibitory concentration of Ceftazidime- Avibactam against Clinical isolates of XDR Salmonella enterica Serovar Typhi
}

Kokab Jabeen ( $\nabla$ kaukab_jabeen@yahoo.com )

University of health sciences, Lahore

\section{Sidrah Saleem}

University of health sciences, Lahore

\section{Summiya Nizam-ud-din}

Shaukat Khanum memorial hospital,Lahore

\section{Faiqa Arshad}

University of health sciences, Lahore

\section{Zill-ehuma}

University of health sciences, Lahore

\section{Shah jahan}

University of health sciences, Lahore

\section{Research Article}

Keywords: azithromycin, Extensively drug resistant typhoid, ceftazidime-avibactam, minimum inhibitory concentration

Posted Date: December 15th, 2021

DOI: https://doi.org/10.21203/rs.3.rs-1169896/v1

License: (c) (1) This work is licensed under a Creative Commons Attribution 4.0 International License. Read Full License 


\section{Abstract}

Background: Typhoid fever is a major healthcare problem in low and middle-income countries. The emergence of extensively drug-resistant (XDR) typhoid has further narrowed down the way to already limited therapeutic options. WHO has listed S.Typhi amongst the priority pathogens against which new treatment options should be discovered.

Objective: To determine In-vitro activity of Ceftazidime-avibactam against clinical isolates of XDR S.Typhi.

Study design: This is a cross-sectional study

Place and duration of the study: The Department of Microbiology, University of health sciences, Lahore from January to June 2021.

Methodology: Antimicrobial susceptibility was performed initially by Kirby Bauer disc diffusion method for 150 of XDR Salmonella enterica Serovar Typhi and MICs of all the recommended antibiotics was determined by VITEK 2 (bioMérieux) fully automated system using Clinical Laboratory Standard Institute (CLSI) 2021 guidelines. MICs by the E-test method were determined for Ceftazidime-avibactam and Azithromycin only. All tests were done in duplicate.

Results: All 150 (100\%) isolates were sensitive to Ceftazidime-avibactam by disc diffusion and E-test methods. Out of 150 isolates 8(5.3\%) were having high MICs against Azithromycin.

Conclusions: Ceftazidime-avibactam can be used wisely to treat ESBL producing XDR typhoid fever cases especially in countries like Pakistan where Typhoid fever is endemic and majority of isolates are extensively drug resistant.

\section{Introduction}

Typhoid fever remains a significant public health threat in low and middle-income countries. According to the most recent estimates, between 11 and 21 million cases and 128000 to 161000 typhoid-related deaths occur annually worldwide [1]. Antimicrobial resistance in Salmonella can be associated with horizontal transference of antibiotic resistant genes characteristically found on mobile genetic elements among Salmonella strains and other Enterobacteria or by clonal spread of antimicrobial drug resistant serovars that are particularly nominated in worldwide dissemination [2]. Since November 2016, a large proportion of ceftriaxone-resistant cases have been identified in the province of Sindh. Similar case was also identified in the United Kingdom from a traveler returning from Pakistan. These S. Typhi strains are resistant to chloramphenicol, ampicillin, trimethoprim-sulfamethoxazole, fluoroquinolones, and thirdgeneration cephalosporins. This new strain has been designated as XDR (Extensively drug resistant) leaving behind azithromycin and carbapenems, the only treatment options [2].This Pakistan outbreak caused by an XDR H58 strain of S.Typhi should be regarded as a clarion call that notifies public health 
authorities globally that we are rapidly approaching a scenario where the acquisition of one more resistance might result in an $S$. Typhi pathogen that is, in practical terms, virtually untreatable in most developing countries.

Now is the time for global action to prevent a gathering storm from becoming a perfect storm and an enormous public health crisis. Ceftazidime is a third generation cephalosporin antibiotic useful for the treatment of a number of bacterial infections. Specifically it is used for joint infections, meningitis, pneumonia, sepsis, urinary tract infections, malignant otitis externa, Pseudomonas aeruginosa infection, and vibrio infection [3]. Like all other third generation cephalosporins, it is active against Salmonella enterica serovars including S. Typhi. But recent emergence of XDR S. Typhi has knocked this drug out of the arsenal because of ESBL production. Avibactam is a non- $\beta$-lactam $\beta$-lactamase inhibitor developed by Actavis.

A new drug application for avibactam in combination with ceftazidime (branded as Avycaz) was approved by the FDA on February 25, 2015, for treating complicated urinary tract (cUTI) and complicated intra-abdominal infections (CIAl) caused by antibiotic resistant-pathogens, including those caused by multi-drug resistant Gram-negative bacterial pathogens [4].Recently approval has been given for bacterial pneumonia and ventilator associated pneumonia caused by multi drug resistant pathogens.

The combination contains the well-established third-generation cephalosporin ceftazidime paired with the novel non- $\beta$-lactam $\beta$-lactamase inhibitor avibactam.Avibactam lacks clinically significant antibacterial activity; however, it inhibits a broad spectrum of $\beta$-lactamases, with high affinity for class $A, C$, and some D enzymes, restoring the in vitro activity of ceftazidime [5]. .

Ceftazidime-avibactam demonstrated potent activity against molecularly confirmed ESBL-producing ( $n=$ 5,354; $\mathrm{MIC}_{90}, 0.5 \mu \mathrm{g} / \mathrm{ml} ; 99.9 \%$ susceptible), plasmid-mediated AmpC-producing ( $n=246 ; \mathrm{MIC}_{90}, 0.5$ $\mu \mathrm{g} / \mathrm{ml} ; 100 \%$ susceptible), and ESBL and AmpC-producing ( $n=152 ; \mathrm{MIC}_{90}, 1 \mu \mathrm{g} / \mathrm{ml} ; 100 \%$ susceptible) isolates of E. coli, K. pneumoniae, K. oxytoca, and P. mirabilis [5].

Ceftazidime/avibactam has been shown to be highly active against Enterobacteriaceae in in-vitro studies, inhibiting a broad spectrum of $\beta$-lactamases. In a study, the activities of ceftazidime-avibactam and comparator antimicrobial agents were tested against 20,709 clinical Enterobacteriaceae isolates collected in U.S. hospitals during the period from 2011 to 2013. Overall, $99.9 \%(20,698$ of 20,709) of Enterobacteriaceae strains were inhibited at a ceftazidime-avibactam MIC of $8 \mu \mathrm{g} / \mathrm{ml}$ or less, which is the ceftazidime-avibactam-susceptible breakpoint. This study included $284 \mathrm{CTX} \mathrm{M}_{\mathrm{M}-15}$ producing isolates and $107 \mathrm{CTX}_{\mathrm{M}-14}$ producers. The potent Gram-negative spectrum of activity of ceftazidime-avibactam, including activity against resistant organisms, demonstrates that it warrants further study in difficult to treat serious infections where resistant gram negative bacteria may occur [6]. Ceftazidime-avibactam activity has been reported in Salmonella enterica having $\mathrm{MIC}_{50 / 90}$ equal to $0.25 / 0.5$ with range of $\mathrm{MIC} \leq$ $0.03-0.5$ and $100 \%$ isolates were sensitive to this combination [7]. 


\section{Study Design}

This was a Cross-sectional study.

\section{Setting}

This study was conducted at The Department of Microbiology, University of Health Sciences, Lahore.

\section{Duration}

6 months (January 2021 to June 2021).

\section{Sample Collection:}

A total of 150 positive blood cultures meeting the inclusion criteria were collected from blood different tertiary care hospitals of Lahore.

\section{Bacterial identification}

The isolates were cultured and purified on Tryptic Soya agar. The blood culture bottle detected as positive was sub-cultured onto Blood agar (Oxoid, UK) and MacConkey agar (Oxoid, UK) incubated at $35-37^{\circ} \mathrm{C}$. The isolate identification was initially performed by Gram-staining. Biochemical identification was done by using Analytical Profile Index-20 Enterobacteriacae system (BioMerieux, France). France) and VITEK2 (bioMérieux) consistent with the manufacturer instructions. S. Typhi was confirmed by agglutination with genus- and serotype-specific antisera (Salmonella poly antiserum A-I [Difco], Salmonella 0 antiserum [Difco], and Salmonella Vi antiserum [Difco].

\section{Antimicrobial Susceptibility}

Antimicrobial susceptibility testing was performed by Standard Kirby-Bauer Disk Diffusion method using cation adjusted Mueller-Hinton agar (MHA) (Oxoid UK), according to Clinical Laboratory Standards Institute guidelines 2021 and zones of inhibition were interpreted according to the breakpoints [8]. The reference strains of E. coli ATCC ${ }^{\circledR} 25922$ and P. aeruginosa ATCC ${ }^{\circledR} 2785$ were used for consistency.

\section{Minimum inhibitory concentration (MIC) determination}

The minimum inhibitory concentration of all the recommended antibiotics was determined by Vitek 2 (bioMérieux) fully automated system. S. Typhi that demonstrated high MICs against ceftriaxone and cefotaxime $(>4 \mathrm{ug} / \mathrm{mL}$ ) was considered as XDR. For Ceftazidime-avibactam MIC was determined by EStrip (liofilchem, Italy) method. A value of $\leq 8 / 4 \mathrm{mg} / \mathrm{L}$ was taken as sensitive according to CLSI 2021 criteria for Enterobacterales [8].

This study was conducted using the bacterial strains which were isolated for diagnostic and treatment purpose. Moreover, the study was completely anonymous, any demographic data or identifiable 
information was not obtained therefore informed consent was not required for such type of study according to the local legislation.

\section{Results}

In this study, a total of 150 XDR S. Typhi isolates were included which were collected over a period of 6 months from different tertiary care hospitals of Lahore.

The antimicrobial susceptibility testing showed that all the Salmonella Typhi isolates were susceptible to Carbapenems (Imipenem \& Meropenem) but 7 isolates had higher Azithromycin MIC and 1 isolate was resistant to Azithromycin (Table 1). The MIC values for Imipenem \& Meropenem were between $\leq 0.06$ to $1 \mu \mathrm{g} / \mathrm{mL}$. Out of 150 isolates 7(4.6\%) were resistant to Piperacillin-tazobactam and rest had MIC between 1 to $\geq 128$. Out of 150 isolates $7(4.6 \%)$ had MIC of $16 \mu \mathrm{g} / \mathrm{mL}$ and only $1(0.6 \%)$ had MIC of $32 \mu \mathrm{g} / \mathrm{mL}$. All the isolates were resistant to Ampicillin, Chloramphenicol, Co-trimoxazole, Ciprofloxacin and Third generation Cephalosporins. For Ceftazidime-avibactam MIC range was between 0.016 to $0.064 \mathrm{mg} /$ $\mathrm{mL}$.Overall 150(100\%) isolates were sensitive to Ceftazidime-avibactam (Table 2).

\section{Discussion}

Typhoid fever is a significant health problem for countries like Pakistan. Emergence and spread of XDR S. Typhi across Pakistan has generated a huge problem for the control and prevention of Typhoid fever. These S.Typhi strains are resistant to chloramphenicol, ampicillin, trimethoprim-sulfamethoxazole, fluoroquinolones, and third-generation cephalosporins. With very limited treatment options already available emergence of Azithromycin resistance would create further problem. There is dire need to look for new therapeutic options as urged by WHO (World health organization).

In this study we explored In-vitro activity of Ceftazidime-avibactam against clinical isolates of XDR S. Typhi collected from different tertiary care hospitals of Lahore. Ceftazidime-avibactam MIC was tested using E-test method against 150 XDR S. Typhi isolates. MIC range was between 0.016 to $0.064 \mathrm{mg} / \mathrm{mL}$. Out of 150 isolates $7(4.6 \%)$ had MIC of $0.016 \mathrm{mg} / \mathrm{mL}, 40(26.6 \%)$ had MIC of $0.023 \mathrm{mg} / \mathrm{mL}, 36(24 \%)$ had MIC of $0.032 \mathrm{mg} / \mathrm{mL}, 37(24.6 \%)$ had MIC of $0.047 \mathrm{mg} / \mathrm{mL}, 22(14.6 \%)$ had MIC of $0.064 \mathrm{mg} / \mathrm{mL}$ and $8(5.3 \%)$ had MIC of $0.094 \mathrm{mg} / \mathrm{mL}$. In our study $150(100 \%)$ isolates were sensitive to Ceftazidimeavibactam.

Ceftazidime-avibactam has shown promising results against MDR and XDR pathogens producing ESBLs in previous studies. In a recent study, a total of 7051 Enterobacterale isolates and 2032 Pseudomonas aeruginosa isolates from hospitalized patients in Australia, Japan, South Korea, Malaysia, the Philippines, Taiwan and Thailand were studied. More than $90 \%$ of all Enterobacterales isolates, including the ESBL-positive, carbapenemase-negative and the carbapenemase-positive, MBL-negative were susceptible to amikacin and ceftazidime-avibactam [9].Ceftazidime-avibactam and comparators were tested by reference broth microdilution against 372 Gram-negative bacilli collected from 11 teaching 
hospitals in China in 2011 and 2012. Avibactam potentiated the activity of ceftazidime against organisms with combinations of ESBLs, AmpCs, and KPC-2 [10].

In another study Ceftazidime-avibactam was tested against 57 well-characterized Gram-negative strains producing beta lactamases from all molecular classes. Avibactam lowered ceftazidime MICs up to 2,048fold against AmpC extended-spectrum beta lactamase (ESBL), and KPC-2 producing Enterobacteriaceae or Pseudomonas aeruginosa [11].

In another study conducted in Spain, Ceftazidime-avibactam was very active against ESBL producing Enterobactarales. Escherichia coli had $\mathrm{MIC}_{90}$ of $0.25 \mathrm{mg} /$ liter and ESBL producing Klebsiella pneumonia had $\mathrm{MIC}_{90}$ of $0.5 \mathrm{mg} /$ liter, Ceftazidime-resistant AmpC-producing species had $\mathrm{MIC}_{90}$ of $1 \mathrm{mg} /$ liter [12].

Furthermore, in a large US focused surveillance program conducted in 2012, ceftazidime-avibactam had antimicrobial activity against $99.8 \%$ of Enterobacteriaceae including those with an ESBL phenotype [12].

Ceftazidime-avibactam can be used as a treatment option in ESBL producing XDR S. Typhi isolates sparing Carbapenems and Azithromycin. This will reserve the use of these drugs in non-responding or difficult to treat cases without significantly creating resistance by overuse. Azithromycin resistance has already been reported from several parts of the world especially typhoid endemic countries like India, Nepal, and Bangladesh [13].Several cases have been reported from Pakistan too [14].

\section{Conclusion}

Azithromycin resistance in XDR S. Typhi is on the rise and it may pose a significant threat to the clinicians especially where XDR S. Typhi is endemic now. Antimicrobial stewardship especially in the post-COVID-19 era is crucial to prevent the development of resistance to this limited oral choice against XDR typhoid. There is vital need to look for new treatment options. Ceftazidime-avibactam has shown promising results in this in-vitro study and it can be a likely candidate for clinical trials.

\section{Declarations}

\section{Ethical approval}

The study was ethically approved by the "Ethical Review Board" under reference number UHS/REG19/ERC/398 from University of health sciences, Lahore, Pakistan.

\section{Conflict of interest}

The authors declare no competing interests

\section{Acknowledgments}

\section{Financial Support}


No funding was involved in the study.

\section{References}

World Health organization, Typhoid, Immunization, vaccines and biologicals, accessed $6^{\text {th }}$ Dec, 2018, https://www.who.int/immunization/diseases/typhoid/en/

Klemm EJ, Shakoor S, Page AJ, Qamar FN, Judge K, Saeed DK, Wong VK, Dallman TJ, Nair S, Baker S, Shaheen G, Qureshi S, Yousafzai MT, Saleem MK, Hasan Z, Dougan G, Hasan R (2018) Emergence of an extensively drugresistant Salmonella enterica Serovar Typhi clone harboring a promiscuous plasmid encoding resistance to fluoroquinolones and third-generation cephalosporins. mBio 9: e00105-18.

Lagacé-Wiens, P., Walkty, A. and Karlowsky, J.A., 2014. Ceftazidime-avibactam: an evidence-based review of its pharmacology and potential use in the treatment of Gram-negative bacterial infections. Core Evid, 9, p.13.

Sader, H.S., Castanheira, M., Flamm, R.K., Mendes, R.E., Farrell, D.J. and Jones, R.N., 2015. Ceftazidime/avibactam tested against Gram-negative bacteria from intensive care unit (ICU) and non-ICU patients, including those with ventilator-associated pneumonia.

Int. J. Antimicrob. Agents, 46(1), pp.53-59.

Karlowsky, J.A., Biedenbach, D.J., Kazmierczak, K.M., Stone, G.G. and Sahm, D.F., 2016. The Activity of Ceftazidime-Avibactam against Extended-Spectrum and AmpC $\beta$-Lactamase-Producing Enterobacteriaceae Collected in the INFORM Global Surveillance Study in 2012-2014. Antimicrob. Agents Chemother, pp.2849-57.

Castanheira, M., Mills, J.C., Costello, S.E., Jones, R.N. and Sader, H.S., 2015. Ceftazidime-avibactam activity tested against Enterobacteriaceae from United States hospitals (2011-2013) and characterization of $\beta$-lactamase producing strains. Antimicrob. Agents Chemother,pp.3509-17.

Li, H., Estabrook, M., Jacoby, G.A., Nichols, W.W., Testa, R.T. and Bush, K., 2015. In vitro susceptibility of characterized $\beta$-lactamase-producing strains tested with avibactam combinations. Antimicrob. Agents Chemother, 59(3), pp.1789-93.

Clinical and Laboratory Standards Institute: Performance standards for antimicrobial susceptibility testing .CLSI Supplement M100. Clinical and Laboratory Standards Institute, Wayne; 2021.

Wen-Chien, K. and Stone, G.G., 2020. In vitro activity of ceftazidime-avibactam and comparators against Gram-negative bacterial isolates collected in the Asia-Pacific region as part of the INFORM program (2015-2017). Ann. Clin. Microbiol. Antimicrob, 19, p.1

Wang, X., Zhang, F., Zhao, C., Wang, Z., Nichols, W.W., Testa, R., Li, H., Chen, H., He, W., Wang, Q. and Wang, $\mathrm{H}$., 2014. In vitro activities of ceftazidime-avibactam and aztreonam-avibactam against $372 \mathrm{Gram}$ - 
negative bacilli collected in 2011 and 2012 from 11 teaching hospitals in China. Antimicrob. Agents Chemother, 58(3), pp.1774-78

Mutters, N.T., Zimmermann, S., Kaase, M. and Mischnik, A., 2015. Activity of temocillin, mecillinam, ceftazidime, and ceftazidime/avibactam against carbapenem-non-susceptible Enterobacteriaceae without carbapenemase production. Eur. J. Clin. Microbiol. Infect. Dis, 34(12), pp.2429-37.

Huband, M.D., Castanheira, M., Flamm, R.K., Farrell, D.J., Jones, R.N. and Sader, H.S., 2016. In vitro activity of ceftazidime-avibactam against contemporary Pseudomonas aeruginosa isolates from United States medical centers by census region (2014). Antimicrob. Agents Chemother, pp.2537-41.

Shoaib, M., Satti, L., Hussain, A., Khursheed, N., Sarwar, S. and Shah, A.H., 2021. Disc Diffusion Testing of Azithromycin Against Clinical Isolates of Typhoidal Salmonellae: A DiagnosticConundrum. Cureus, 13(7).

Iqbal, J., Dehraj, I.F., Carey, M.E., Dyson, Z.A., Garrett, D., Seidman, J.C., Kabir, F., Saha, S., Baker, S. and Qamar, F.N., 2020. A race against time: reduced azithromycin susceptibility in Salmonella enterica serovar Typhi in Pakistan. MSphere, 5(4), pp.e00215-20.

\section{Tables}

Table: 1 MIC distribution of S.Typhi isolated from blood cultures

\begin{tabular}{|l|l|l|l|l|l|l|l|l|l|l|l|l|l|l|}
\hline \multicolumn{9}{|c|}{ No. of isolates having MICs in $\mu$ /mL } & \multicolumn{1}{l|}{} \\
\hline $\begin{array}{l}\text { Antibacterial } \\
\text { Agent }\end{array}$ & Breakpoint & $\begin{array}{l}\leq \\
\mathbf{0 . 0 6}\end{array}$ & $\mathbf{0 . 1 2 5}$ & $\mathbf{0 . 2 5}$ & $\mathbf{0 . 5}$ & $\mathbf{1}$ & $\mathbf{2}$ & $\mathbf{4}$ & $\mathbf{8}$ & 16 & 32 & $\mathbf{6 4}$ & $\begin{array}{l}\geq \\
128\end{array}$ & Total \\
\hline Ampicillin & $\geq 32$ & 0 & 0 & 0 & 0 & 0 & 0 & 0 & 0 & 0 & 0 & 95 & 55 & 150 \\
\hline $\begin{array}{l}\text { Piperacillin- } \\
\text { tazobactam }\end{array}$ & $\geq 128 / 4$ & 0 & 0 & 0 & 8 & 12 & 10 & 45 & 33 & 2 & 13 & 20 & 7 & 150 \\
\hline Ceftriaxone & $\geq 4$ & 0 & 0 & 0 & 0 & 0 & 0 & 0 & 30 & 42 & 35 & 15 & 28 & 150 \\
\hline Cefixime & $\geq 4$ & 0 & 0 & 0 & 0 & 0 & 0 & 0 & 38 & 15 & 48 & 29 & 20 & 150 \\
\hline Cefotaxime & $\geq 4$ & 0 & 0 & 0 & 0 & 0 & 0 & 0 & 20 & 12 & 48 & 33 & 37 & 150 \\
\hline Cefepime & $\geq 16$ & 0 & 0 & 0 & 0 & 0 & 0 & 0 & 0 & 0 & 31 & 73 & 46 & 150 \\
\hline Imipenem & $\geq 4$ & 0 & 30 & 28 & 80 & 12 & 0 & 0 & 0 & 0 & 0 & 0 & 0 & 150 \\
\hline Meropenem & $\geq 4$ & 5 & 13 & 32 & 55 & 45 & 0 & 0 & 0 & 0 & 0 & 0 & 0 & 150 \\
\hline Azithromycin & $\geq 32$ & 0 & 0 & 0 & 0 & 0 & 92 & 50 & 0 & 7 & 1 & 0 & 0 & 150 \\
\hline
\end{tabular}

*The MIC value of Piperacillin-tazobactam are expressed as MIC values of Piperacillin with equal $(4 \mu \mathrm{g} / \mathrm{mL})$ concentration of tazobactam

Table: 2 MIC of CTZ-AVI* for XDR S.Typhi 


\begin{tabular}{|l|l|l|l|}
\hline MIC $(\mathrm{mg} / \mathrm{mL})$ & $\mathrm{n}(\%$ isolates) & $\mathrm{MIC}(\mathrm{mg} / \mathrm{mL})$ & $\mathrm{n}(\%$ isolates) \\
\hline 0.016 & $07(4.6 \%)$ & 3 & $00(0 \%)$ \\
\hline 0.023 & $40(26.6 \%)$ & 4 & $00(0 \%)$ \\
\hline 0.032 & $36(24 \%)$ & 6 & $00(0 \%)$ \\
\hline 0.047 & $37(24.6 \%)$ & 8 & $00(0 \%)$ \\
\hline 0.064 & $22(14.6 \%)$ & 12 & $00(0 \%)$ \\
\hline 0.094 & $08(5.3 \%)$ & 16 & $00(0 \%)$ \\
\hline 0.125 & $00(0 \%)$ & 24 & $00(0 \%)$ \\
\hline 0.19 & $00(0 \%)$ & 32 & $00(0 \%)$ \\
\hline 0.25 & $00(0 \%)$ & 48 & $00(0 \%)$ \\
\hline 0.38 & $00(0 \%)$ & 64 & $00(0 \%)$ \\
\hline 0.50 & $00(0 \%)$ & 96 & $00(0 \%)$ \\
\hline 0.75 & $00(0 \%)$ & 128 & $00(0 \%)$ \\
\hline 1.0 & $00(0 \%)$ & 192 & $00(0 \%)$ \\
\hline 1.5 & $00(0 \%)$ & 256 & $00(0 \%)$ \\
\hline 2 & $00(0 \%)$ & & \\
\hline
\end{tabular}

* The MIC value of Ceftazidime-avibactam are expressed as MIC values based on dosage regimen of $2.5 \mathrm{~g}(2 \mathrm{~g}$ Ceftazidime and $0.5 \mathrm{~g}$ of Avibactam) 8 hourly 